\title{
AN EXPERIMENTAL INVESTIGATION AND FINITE ELEMENT ANALYSIS OF COMPOSITE ENERGY ABSORPTION SYSTEMS
}

\author{
Hakim S. Sultan \\ Mechanical and Manufacturing Engineering Department, Faculty of Engineering, University Putra \\ Malaysia, 43400 UPM, Serdang, Selangor, Malaysia \\ hakimss@hotmail.com
}

Polymer matrix fiber-reinforced composite materials offer many advantages over conventional materials for structural applications, particularly where weight is an important aspect of the design. Recent work has shown that structures made from these materials are capable of absorbing large amounts of energy in a controlled manner. The specific energy absorption coefficient of polymer matrix composites in tubular form is much greater than for conventional metallic materials. Thus, composites are candidate materials for applications in transport where lightweight structures are required which provide a measure of protection in overload impact situations. Work on the axial compression of axisymmetric tubes, to be used in energy absorbing applications, has been extended to cones.

In this paper, experimental and finite element results for the composite conical shell subjected to quasi static axial loading and slipping solid steel cone into composite cone are discussed. The effects of semi-vertex angle of conical shell on the crushing behaviour and energy absorption capabilities were investigated for woven roving glass/epoxy subjected to quasi-static loading are presented and discussed. The semi-vertex angles of the cone used were 4, 8, 12, 16 and 20 degrees. The cone height and bottom diameter were kept constant for all cases as $100 \mathrm{~mm}$ and $76.2 \mathrm{~mm}$, respectively. Experimental and finite element simulation was designed to predict the loaddisplacement curves, deformation histories and energy absorption capability throughout the Eglass fibre fabricated. Detailed discussion on the effects of semi-vertex angle on the crushing behaviour of conical shells and energy absorption capability are discussed. The effect of semivertex angle of conical composite tube on load carrying capacity; in which the specimen composed of one part with same thicknesses from woven roving glass/epoxy composite laminate and tested under quasi-static axial load and slipping solid steel cone into composite cone. The results are presented in terms of above-defined parameters, such as specific energy absorption, volumetric energy absorption, crush force efficiency, initial failure indicator, strain efficiency and failure modes. Details of these crashworthiness parameters are presented and discussed. From these tests it is seen that the force-displacement behaviour shows a polynomial relationship prior to the first crush initiation, The results demonstrated that at first crush stage the energy is dissipated in the form of friction and crazing and the conical responded in an elastic manner, while the post crush stage is dominated by tearing failure mechanisms. The experimental work was designed to predict the load-displacement curves, deformation histories and energy absorption capability throughout the E-glass fiber fabricated and semi-vertex angles. The relations between load and displacement for the composite cones and then calculate the crashworthiness parameters and energy absorption capabilities are presented. An encouraging agreement was obtained between the experimental results of composite conical shell and the numerical predictions.

\section{Keywords:}

Conical Shell, semi-vertex angle, energy absorption 